\title{
Precise Control of Dynamic Object by Combining of the Advantages of Different Sensors
}

\author{
Vadim Zhmud*, Oleg Yadrishnikov and Vladimir Semibalamut
}

Novosibirsk State Technical University and Geophysical Service of SB RAS, Russia

\begin{abstract}
Precise control of dynamic objects with negative feedback loop is widely used in science and industry [1-3]. Control accuracy depends on properties of the regulator, as well as on the accuracy of the sensor of the output value. Often the choice of the best sensor is impossible, because the different sensors may have different, and none of them is the best over the whole frequency range. In this paper, the research work is carried out in the base of the mathematical simulation and some theory. The modeling proof of the method effectiveness is carried out on specific examples. The sensors together with the object give the task with single input and two outputs (SITO).
\end{abstract}

Keywords: Control, feedback, automation, regulator, quality of control, precision of control.

\section{INTRODUCTION}

Control of objects with negative feedback loop ensures high accuracy in the presence of disturbances, so it is widely used. Stabilization systems require that the output value of the object would not changed during the time, despite the fact that an object is affected by some disturbance tending to change this output value. In such systems, error of the control or stabilization $\mathrm{e}(\mathrm{t})$ is equal to the difference between the prescribed output signal of the object $\mathrm{v}(\mathrm{t})$ and the actual output value $y(t)$. This error signal is converted into a control signal $u(t)$, which is applied to the input of the object. The result of this allows achieving of the desired signal equation $y(t) \approx v(t)$, at least on average, (inside a limited bandwidth). If $v(t)=0$, then the feedback is used to stabilize the output value of an object with high accuracy. Such systems must have particularly high accuracy. In some cases the higher is the accuracy of the stabilization, the better it is. Examples of such systems can serve the system for the stabilization of the frequency or phase of the laser radiation for super stable optical frequency standard [1-3]. The monograph [4] has proposed the use of two sensors to combine their advantages and overcome their weaknesses. It is important to calculate the regulator so that advantages of the sensors would be combined indeed, and the disadvantages would be removed. The principle of this approach is to use in the different frequency bands namely those sensors whose accuracy is higher in these ranges than this of other ones. It is necessary also to resolve the problems of pairing of these areas and the calculation of regulators, providing the system stability and its required quality of the control or stabilization.

*Address correspondence to this author at the Novosibirsk State Technical University and Geophysical Service of SB RAS, Russia; Tel: +7-961-875-1917; Fax: +7-383-3461119; E-mail: oao_nips@bk.ru
This paper explores the proposed method by mathematical modeling and numerical optimization of the regulators. The verification of the method has been performed at an example.

\section{STATEMENT OF THE PROBLEM}

Let there are more than one sensor of the output value of the object. First, let consider the situation with the two sensors. Suppose the first sensor has higher signal-to-noise ratio (SNR) in the high-frequency region, and the second one has it higher in the lowfrequency region. The task is to design the regulator, which would ensure controlling of the object in accordance with such SNR, which corresponds to the best one of the sensors in the given frequency domain.

The obvious variant of sensors switch that one can offer on the base of the most common reasons, is to connect all the sensors in parallel to the object, for the measurement of its output value. At the outputs of the sensor, one should use equalizing filters, which would provide a proper combination of the output signals of the sensors with the necessary weight gains. The corresponding block diagram is shown in Figure 1. This scheme is not recommended due to a specific problem. In this Figure $\mathbf{1}$ there are two sensors of the same value $Y(t)$. Coordination of the transfer functions of these sensors is a complex task. Its solution with the alternative approach is not required. Therefore, the circuit shown in Figure $\mathbf{1}$ is not recommended for use, although it is quite clearly demonstrates the principle of the system with two sensors.

Let us consider the structure shown in Figure 2. There Laplace transform of a function of time are marked in large letters, and initial time-varying functions are indicated with small letters. For example, 
$Y(s)$ is the conversion of the output value $y(t), N_{k}(s)$ is the transformation from $k$-th noise sensor $n_{k}(t), Q_{k}(s)$ is the output signal of the $k$-th sensor $q_{k}(t), E_{k}(s)$ is the conversion of the error calculated by the signal from the k-th sensor $\mathrm{e}_{\mathrm{k}}(\mathrm{t})$, and so forth.

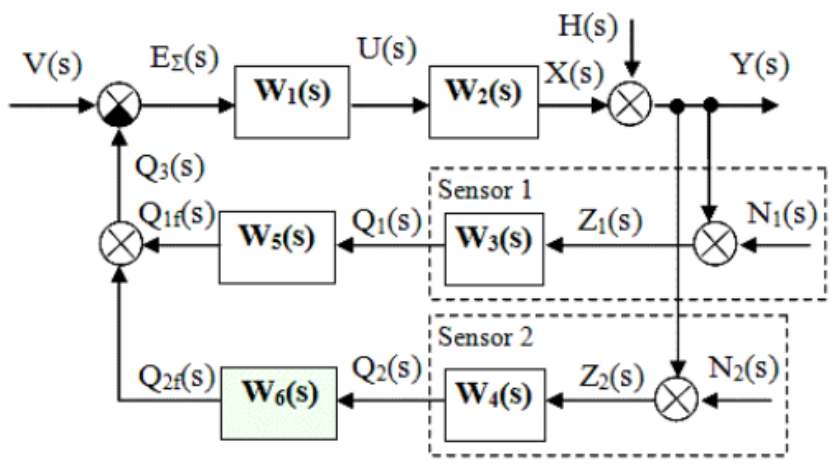

Figure 1: Not recommended structure of the system with two sensors.

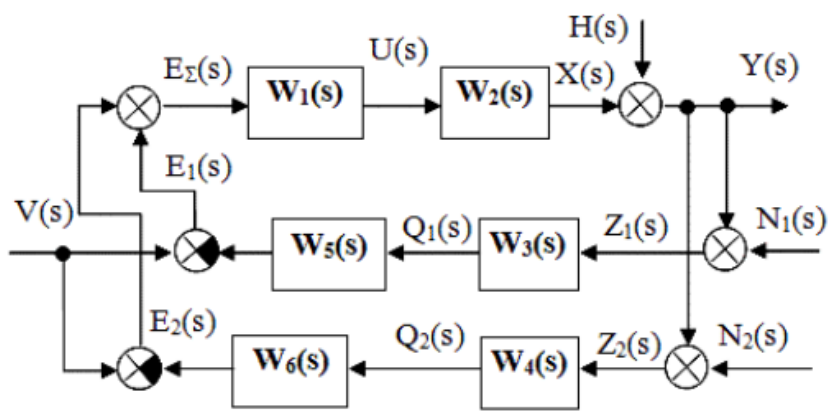

Figure 2: Not recommended structure of the system with two sensors.

In this scheme, the value of error $\mathrm{e}(\mathrm{t})$ of the output signal $y(t)$ is calculated separately based on the output signals from the two sensors: $q_{1}(t)$ and $q_{2}(t)$. Each of the obtained errors $e_{1}(t)$ and $e_{2}(t)$ must be zero. If the feedback signal equal to the sum of these errors is fed to the object through the gain controller $W_{1}$, then the total error is reduced to zero. For correct operation of this loop it requires no coordination of the scope of the transfer functions $W_{3}$ and $W_{4}$. In addition, it is not required to receive signals output estimation value gradually complement each other at the intermediate frequency range. If both sensors are ideal even in the absence of filters, i.e. when $W_{3}=W_{4}=1$, we will get $N_{1}=N_{2}=0$, hence we will obtain $e_{\Sigma}(t)=2 e(t)$. This is equivalent to an increase of the coefficient of the regulator twice. If the system remains stable, it works correctly. The error will be reduced to zero. Therefore, the output signal in the operating frequency range with a desired precision repeats the reference signal $y(t) \approx v(t)$.
For the convenience, we assume that the filter transfer function $W_{3}$ and $W_{4}$ complete the transfer functions of the sensor to the unit in the field of operating frequencies. If it is not so, then the according gain coefficient is necessary. Let combine these transfer functions with the sensor transfer function: $\mathrm{W}_{3}(\mathrm{~s}) \cdot \mathrm{W}_{5}(\mathrm{~s})=\mathrm{W}_{7}(\mathrm{~s}) ; \mathrm{W}_{4}(\mathrm{~s}) \cdot \mathrm{W}_{6}(\mathrm{~s})=\mathrm{W}_{8}(\mathrm{~s})$. The transfer function of the serial regulator, on the contrary, is divided into two parallel transfer functions $W_{9}(\mathrm{~s})$ and $\mathrm{W}_{10}(\mathrm{~s})$. Hence, we obtain a system with two sensors and two regulators, as shown in Figure 3.

In this case, the formulation of the problem is as follows.

Task 1. Two transfer functions of the two sensors are given, which differ so that none of them is the best in the entire operating band. Furthermore, both sensors have noises, the characteristics of which are different and are given initially. It is required to calculate the regulator, which would ensure the highest accuracy of the stabilization (or control) of the output value of the object.

Task 2. Expansion of the Task 1 for the case of three or more sensors.

Note. Because of the linearity of the system and of the absence of any other links from the reference signal $\mathrm{v}(\mathrm{t})$ into the control loop, except the main one thought the subtracting elements calculating the error of the control, the task of stabilization and the task of the control can be solved identically. The result of their decision can be quite revealing described by the response to a step jump disturbance $h(t)$ or stepwise jump of the prescribed value $v(t)$.

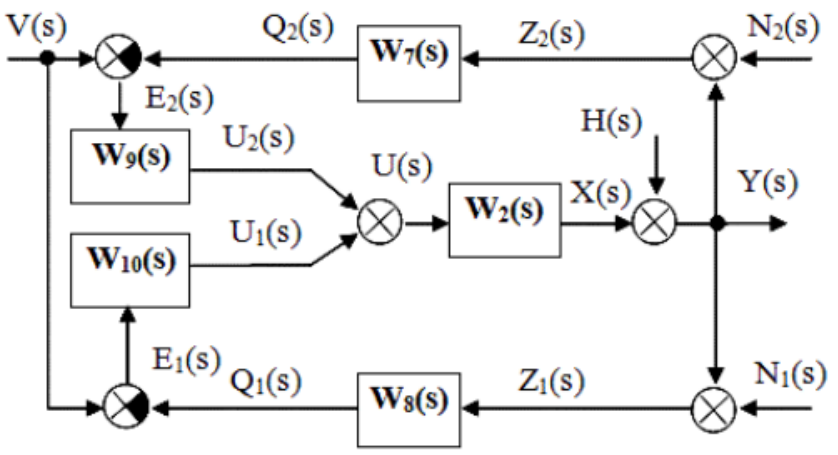

Figure 3: Structure of the system with two sensors.

Let us consider three different sensors. Their mathematical models and appropriate responses to the harmonic signal are shown in Figure 4, where the modeling program VisSim 6.0 has been used. 
For simplicity, we assume a static coefficient of all sensors to be unit. If it is not so, it is necessary to introduce sequentially connected scale amplifier with the corresponding gain. If the digital signals are processed then the equalizing coefficient is necessary.

Let the transfer function of the first sensor has the form of first order filter with time constant $T_{1}=0.5 \mathrm{~s}$. Also in this model, there is a constant offset of the sensor output by the value of 0.4 , and there is additive highfrequency noise (Gaussian noise). The model of this sensor is shown in Figure $\mathbf{4}$ at the top of the figure connected to the input of the oscilloscope with top (red) plotter, a corresponding output signal when the input harmonic signal of this sensor is shown with a strongly oscillating (red) line on the graph Figure 4.

Let the transfer function of the second sensor has the form of a first-order element with a time constant $\mathrm{T}_{2}=10 \mathrm{~s}$. The corresponding model is shown in the middle of Figure $\mathbf{4}$ and the output signal is shown with slightly deviating (blue) line.

Let the model described by the third sensor transfer function is first-order filter with a time constant $T_{3}=0.05 \mathrm{~s}$. Also to the output signal of the sensor is mixed with slowly changing harmonic signal with a period of $100 \mathrm{~s}$. The corresponding model is in the bottom of Figure 4, and the output signal is the middle (black) line.

\section{THE RESULTS OF OPTIMIZATION OF THE SYSTEM WITH VARIOUS SENSORS}

In our paper [4] we has proposed to choose most fast sensors for the derivative link and sensors with a higher static accuracy for integrating link, which is quite logical. This assertion can be developed by proposition that for each frequency range it is better to use the sensor that has the best SNR in this area.

In addition to theoretical studies, we propose the research based on mathematical modeling. The proposed methodology of this study is as follows.

1. Let us select the sensors with the appropriate models and on the basis of their output signals create the structure with negative feedback, for example, PID-regulator, i.e. the regulator containing the proportional, integrating and derivative links.

2. The coefficient of each link should be optimized automatically, if we set the cost function. We define, for example, the cost function as an integral of the module of error multiplied by the time from the beginning of the transient process, which is initiated by a single step jump.

3. We repeat the above procedure to for various combinations of the sensors.

4. At least we compare the results with the various sensors using transient processes or the cost function. On this base we develop the according conclusions.

One of the logical ways of the choices of sensor is the choice of the sensor number 2, ignoring the possibility of using other sensors. The basis for this choice is the fact that this sensor contains in it model only a first-order filter. In this sensor, there is no noise

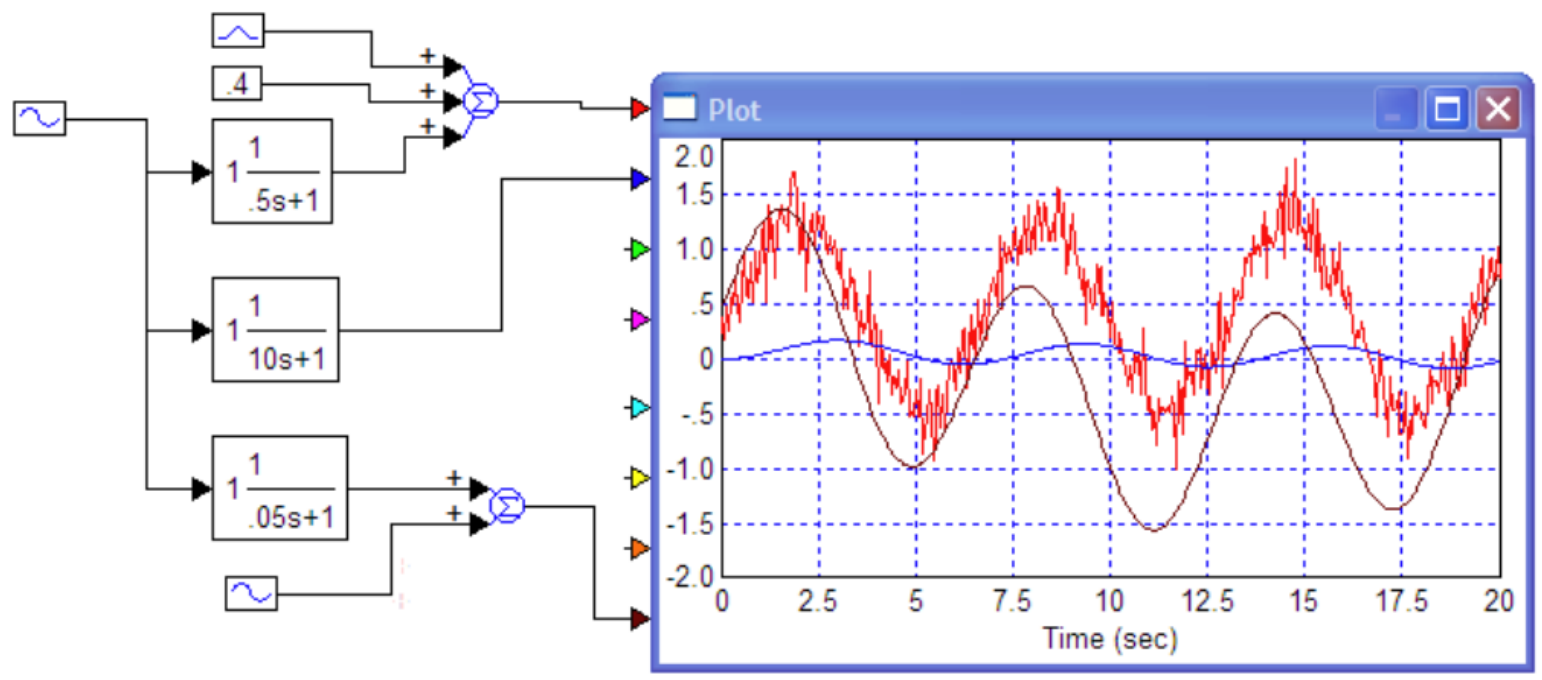

Figure 4: Block diagram of the three sensors and the simulation result in the program VisSim 6.0. 


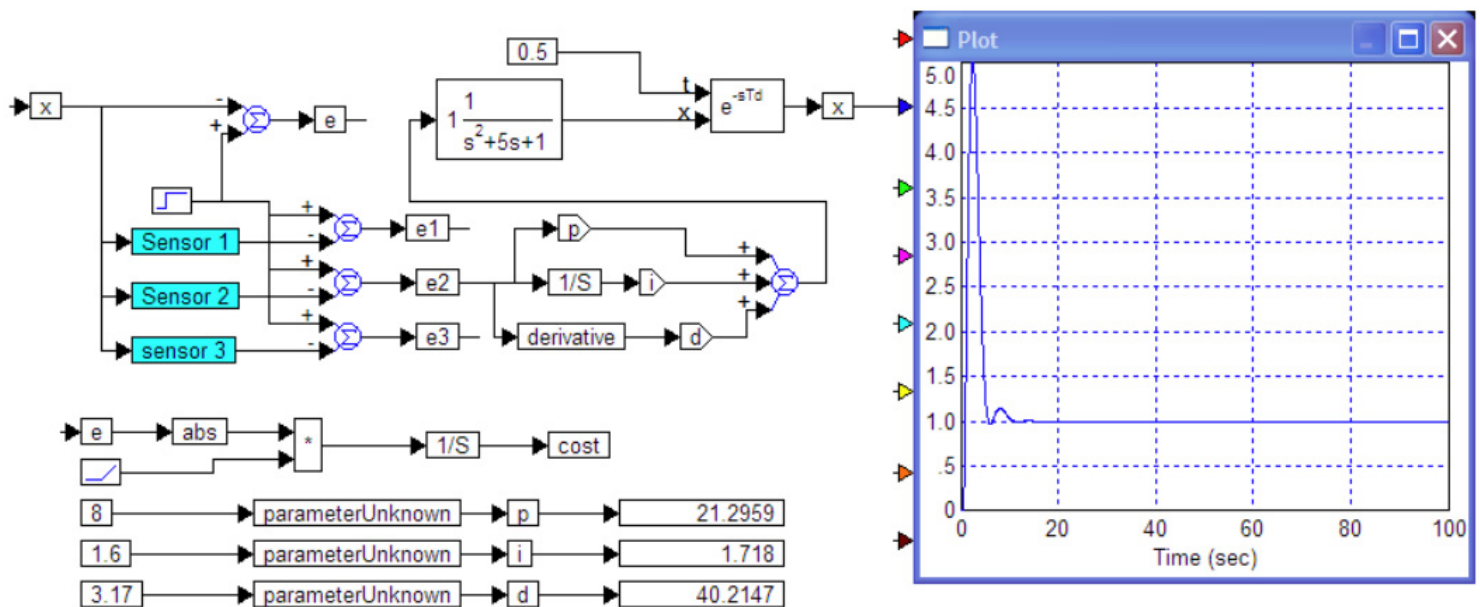

Figure 5: The results of the optimization of the system with only second sensor.

or zero shifts. The results from the optimization of systems with the only this sensor is shown in Figure $\mathbf{5}$. Here the overshoot reaches 5 units, i.e. $400 \%$. Such a system can not be considered acceptable. The duration of the transient process is approximately $10 \mathrm{~s}$.

Let consider the results of using only the third sensor which are shown in Figure 6.

The results of using only the third sensor also can not be declared admissible, since the duration of the transient process exceeds 100 , the overshoot is about $30 \%$.

The results of using only the first sensor is not required to simulate and analyze, it is obvious that in this case, a static error will be 0.4 units (for the unit step it is $40 \%$ ).

On the basis of preliminary considerations on the SNR for different frequency regions we can offer the following variant of the use of the sensors: for proportional link we propose the use of the first sensor, for the integral link the use of the second sensor, and for the derivative link the use of the third sensor. Such a structure and its optimization results are shown in Figure 7.

The optimization results are shown in Figure 7 too. Although these results are better of the other previous results from the point of view of some parameters, the transient process is still not satisfactory. Indeed, the overshoot decreased to a value of no more than $10 \%$, the transient process as a whole ends after 40 seconds, but the result of the control is characterized by unacceptably high levels of noise, in particular: the peak deviation of the output value from the specified value reaches $10 \%$. In fact, one could argue that the transient process does not end there, because the system has to work (repeat) sensor noise.

The result of using of only two sensors is shown in Figure 8. In this case, the second sensor is also used

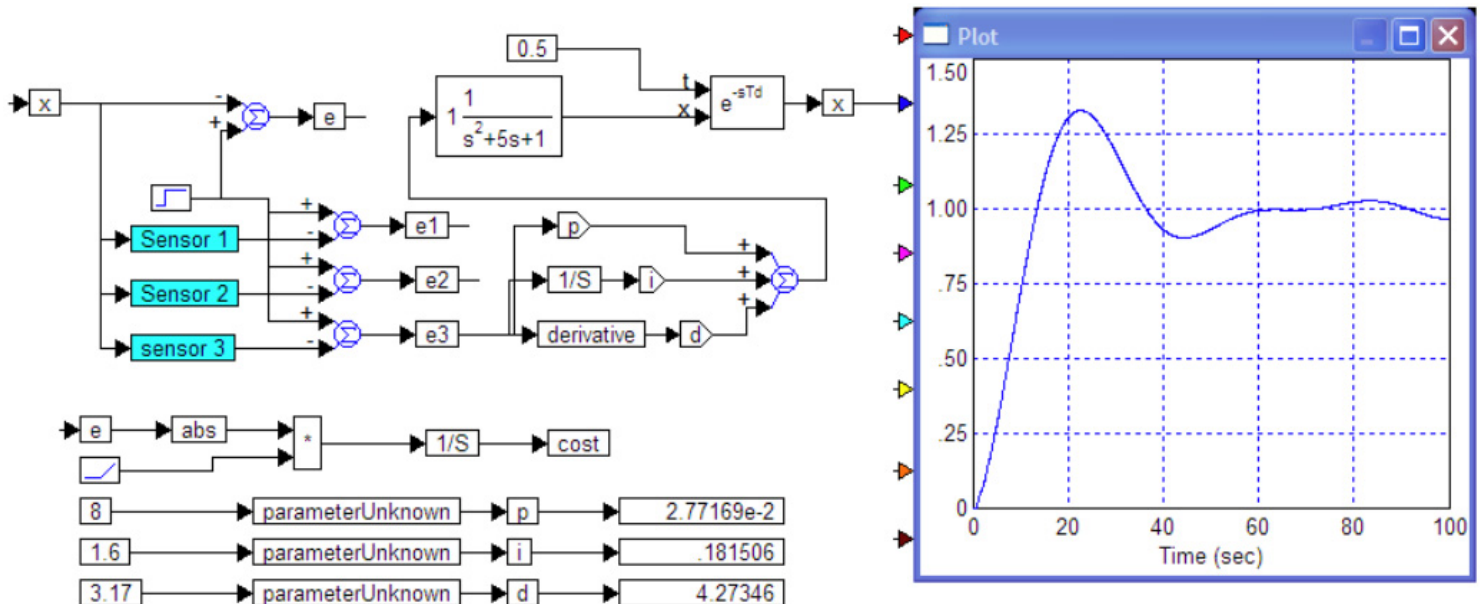

Figure 6: The results of the optimization of the system with only third sensor. 


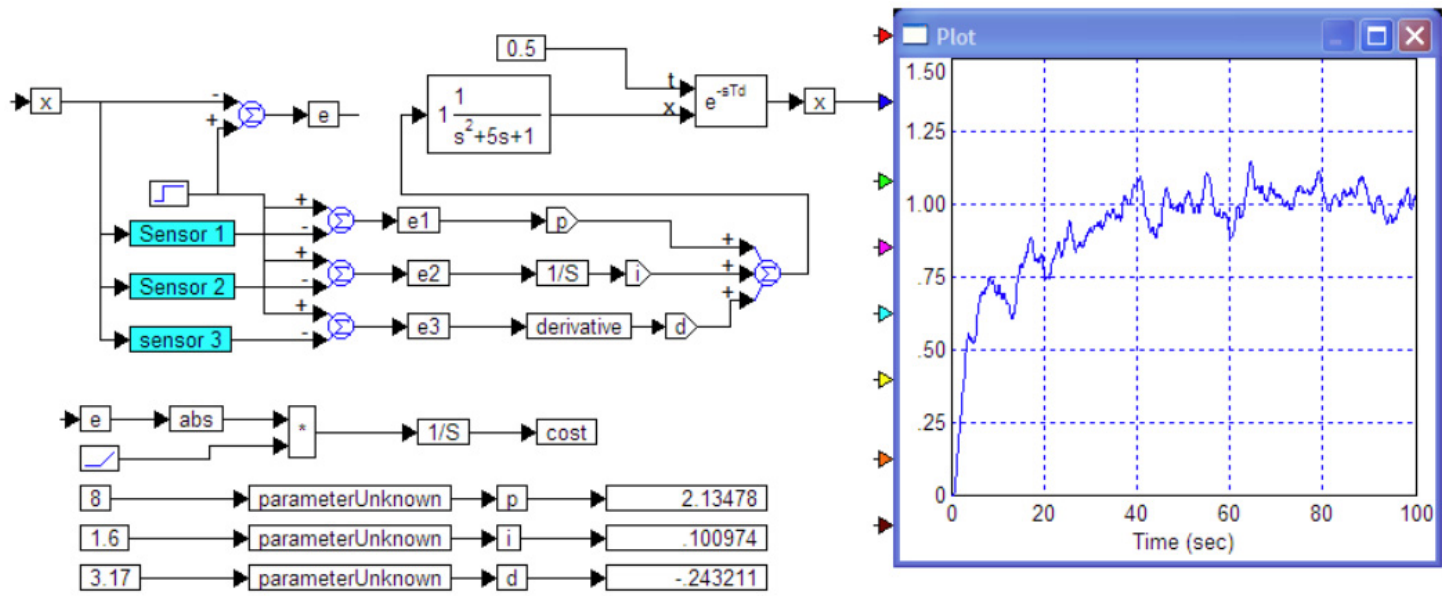

Figure 7: The results of the optimization of the system using the three sensors.

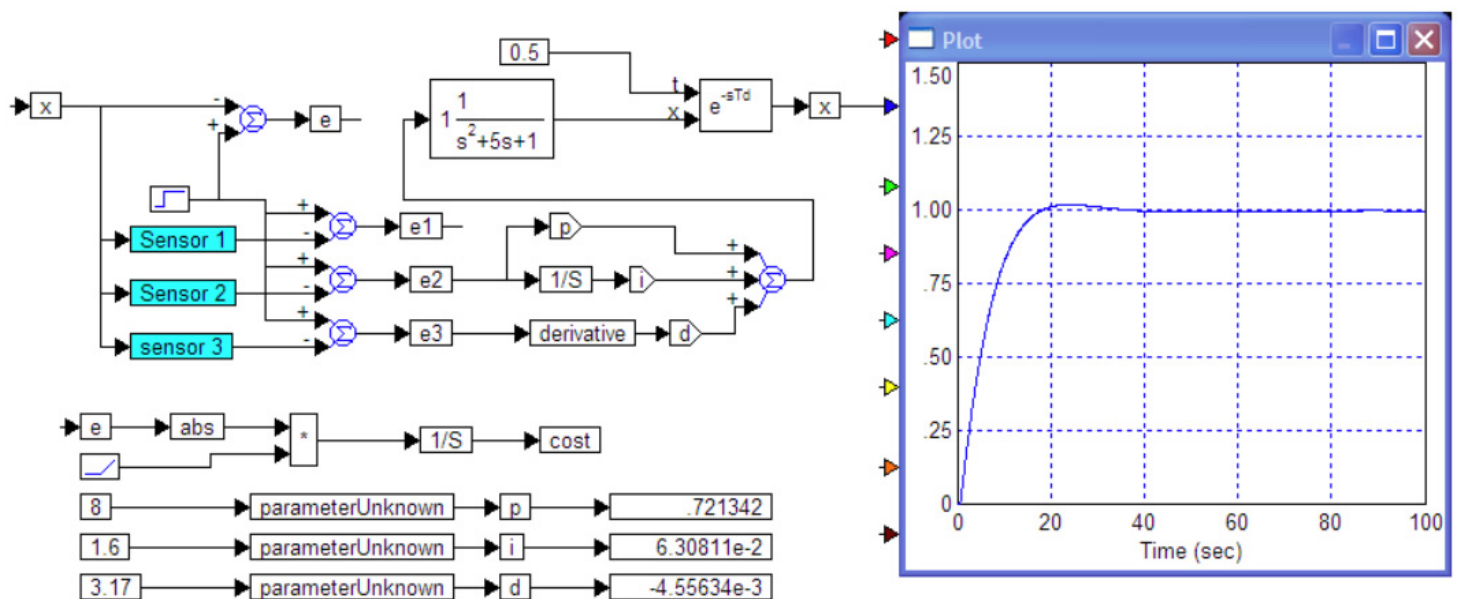

Figure 8: The results of the optimization of the system using the second and the third sensors.

both for proportional and for integrating links, and the third sensor is used for the derivative link. Here the first sensor is not used.

The result shown in Figure 8 is definitely the best of all possible results. Indeed, the transient process ends after $20 \mathrm{~s}$ from the start. The overshoot is negligible (about $1 \%$ ), and the output signal has no noises.

Thus, the numerical optimization of the regulator allowed on the basis of a formal procedure to eliminate the excess sensor from the system structure, which has led to a simplification. It also allowed using the remaining sensors in the best possible combination with the best choice of the coefficients of the regulator owing the optimizing.

This investigation has demonstrated the following:

1. The use of several sensors of the same value, provided that they are characterized by different properties (for speed and (or) at the level of noise) allows to achieve the best quality control, the result of this calculation can be made efficiently in the case of automatically numerical optimizing of the regulator.

2. Not every sensor, which on the basis of general considerations may seem to be useful is really useful. Numerical optimization procedure showed that the use of some sensors it is advisable to give up.

\section{CONCLUSIONS}

The paper have studied the method of calculating of the two-channel regulator of one output variable based on numerical optimization using many sensors of the output value. The feasibility and effectiveness of using more than one sensor is revealed and confirmed with the simulation. Also, the feasibility of not using of some sensor is revealed. This is not obvious from general considerations of the sensors models. 
This work was financially supported by the Russian Ministry of Education and Science, project number $2014 / 138$

\section{REFERENCES}

[1] Zhmud V, Yadrishnikov O. Numerical optimization of PIDregulators using the improper moving detector in cost function. Proceedings of the 8-th International Forum on Strategic Technology 2013 (IFOST-2013), vol. II, 28 June - 1 July. Mongolian University of Science and Technology, Ulaanbaator, Mongolia. IEEE organized 2013; pp. 265-270. http://www.must.edu.mn/IFOST2013/

[2] Zhmud V, Dimitrov L. Designing of complete multi-channel PD-regulators by numerical optimization with simulation
2015. 2015 International Siberian Conference on Control and Communications, SIBCON 2015 - Proceedings, 7147059, 10.1109/SIBCON.2015.7147059, http://www.scopus.com inward/record.url?eid=2-s2.0-84941057726\&partnerlD= 40\&md5 $=6 \mathrm{~b} 22 \mathrm{ccb} 4 \mathrm{fc} 8 \mathrm{da} 0 \mathrm{~d} 7 \mathrm{cb} 570 \mathrm{eb} 23 \mathrm{~d} 62 \mathrm{fcfb}$, Conference Paper, Scopus, 2-s2.0-84941057726

[3] Zhmud VA, Yadrishnikov OD, Semibalamut VM. Control of the objects with a single output and with two or more input channels of influence. WIT Transaction on Modelling and Simulation. WIT Press. www.witpress.com, ISSN 1743-355X (on-line). 2015; Vol. 59: pp. 147-156.

[4] Zhmud VA. Simulation, research and optimization of locked system of automatic control. Monograph. Novosibirsk (In Russian). Publishing house of NSTU 2012; p. 335. ISBN 978-5-7782-2162-8.

(C) 2015 Zhmud et al.; Licensee Lifescience Global.

This is an open access article licensed under the terms of the Creative Commons Attribution Non-Commercial License (http://creativecommons.org/licenses/by-nc/3.0/) which permits unrestricted, non-commercial use, distribution and reproduction in any medium, provided the work is properly cited. 